\title{
Erratum: Structural Origin of Enhanced Dynamics at the Surface of a Glassy Alloy [Phys. Rev. Lett. 119, 245501 (2017)]
}

Gang Sun, Shibu Saw, Ian Douglass, and Peter Harrowellø

(Q) (Received 3 September 2020; published 25 September 2020)

DOI: 10.1103/PhysRevLett.125.139901

A textual error appears in this Letter that incorrectly describes how the participation fraction $f_{\alpha}$ of the $\alpha$ th normal mode in the glass surface was calculated. The correct expression for this quantity is $f_{\alpha}=\left(\sum_{j}^{N_{s}} v_{\alpha, j}^{2} / \sum_{j}^{N} v_{\alpha, j}^{2}\right)$, where $v_{\alpha, j}^{2}$ is the squared amplitude of the contribution of particle $j$ to the eigenvector of the $\alpha$ th normal mode and the sum $\sum_{j}^{N_{s}} v_{\alpha, j}^{2}$ is over the $\mathrm{N}_{s}$ surface atoms only. All numerical results presented are correctly calculated and are unaffected by this textual error. 